\title{
Carcinoma de Endometrio. Quince años de experiencia
}

\author{
HOSPITAL UNIVERSITARIO SAN VICENTE DE PAUL \\ MEDELLIN - COLOMBIA \\ Dres.: Gildardo Gallego Noreña*, Jaime Uribe Duque**, \\ Juan Luis Londoño***
}

\section{INTRODUCCION}

El Cáncer Endometrial ocupa el tercer lugar en frecuencia entre las neoplasias genitales malignas de la mujer. Las publicaciones a nivel mundial indican un incremento en la frecuencia, en oposición al cáncer cérvico uterino que muestra una franca disminución.

A nivel nacional las publicaciones sobre cáncer endometrial son escasas (1, 2) y por esta razón, es difícil seguir la tendencia de esta neoplasia en nuestro medio.

Creemos que en el Grupo de Oncología Ginecológica del Hospital Universitario San Vicente de Paúl, el número de casos registrados durante quince años es considerable. Vemos urgente la necesidad de registrar esta neoplasia de

\footnotetext{
* Profesor Asociado 750 unidades.

** Profesor Titular Ad-Honorem

**** Profesor Salu Pública

Universidad de Antioquia.
}

acuerdo a un protocolo de estudio, donde se consignen factores predisponentes, epidemiológicos y etiológicos, así como también patrones de tratamiento unificado siguiendo las normas terapéuticas a nivel mundial.

Motivados por estas inquietudes se realiza esta revisión, que aunque es retrospectiva, nos permita hacer un diagnóstico del estado actual de esta neoplasia en el Hospital Universitario San Vicente de Paúl de Medellín. También permitirá ver y analizar las principales fallas en el manejo de esta entidad $y$ basado en los resultados obtenidos, organizar un estudio prospectivo, que tienda a eliminar fallas en el tratamiento, registro y seguimiento de esta entidad.

El promedio de vida de la mujer es mayor en este momento, lo que se traduce en un incremento del cáncer endometrial. Al ubicarnos en países del Tercer Mundo o pa ís poco industrializado presumimos que el índice de cáncer endometrial es bajo en relación con pa íses industrializados. 


\section{OBJETIVOS DEL PROYECTO}

\section{1. Óbjetivo General}

Conocer la frecuencia del cáncer de endometrio en las pacientes remitidas a la sección de Oncología Ginecológica del Departamento de Obstetricia y Ginecología de la Facultad de Medicina de la Universidad de Antioquia.

1.2. Iniciar un protocolo prospectivo para unificar el manejo y tratamiento, además, mejorar sobrevida.

\section{OBJETIVOS ESPECIFICOS}

2.1. Conocer el número total de pacientes con cáncer endometrial en estos quince años.

2.2. Conocer la frecuencia del cáncer endometrial por grupos etáreos.

2.3. Establecer en número de pacientes por estadios de acuerdo con la clasificación clínica.

2.4. Conocer la frecuencia del tipo histológico predominante en el cáncer de endometrio.

2.5. Buscar el tipo de tratamiento realizado.

2.6. Conocer el seguimiento de las pacientes con cáncer de endometrio y como consecuencia tratar de sacar tablas de vida.

2.7. Averiguar el estudio patológico de la muestra en las pacientes que fueron intervenidas y ver la persistencia del tumor.

\section{MATERIAL Y METODOS}

Se revisaron 122 historias clínicas de las pacientes que asistieron al servicio de Oncología Ginecológica con diagnóstico de carcinoma endometrial comprobado por anatomía patológica.

Las pacientes están registradas en computador desde el año 1970 hasta el momento actual. Esta revisión se hace desde el 1o. de enero de 1970 hasta el 30 de junio de 1985.

La evaluación de las pacientes se ha realizado en las consultas llamadas Comité de Oncología que funciona martes y viernes de cada semana y las consultas de seguimiento oncológico que se realiza tres días semanalmente.

\section{RESULTADOS}

Gráfica No. 1

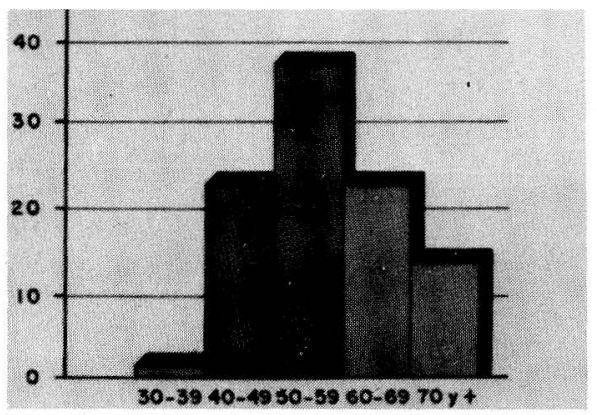

En esta gráfica se analiza la distribución por edad: encontramos que el mayor número de pacientes está en la dي́cada de 50 a 59 años. Sin embargo, también hay un número considerable de casos: $22.9 \%$ en las décadas de 40-49 y 60-69 respectivamente, lo que está de acuerdo con los hallazgos de la literatura médica que habla de mayor incidencia en mujeres perimenopaúsicas $\mathrm{V}$ postmenopáusicas. Las dos pacientes ubicadas en la década de 30 a 39 años tenían específicamente 36 años y 33 años siendo ésta la paciente más jóven registrada con cáncer de endometrio. En la literatura se ha informado pacientes tan jóvenes como el caso de una de 
16 años (Hertig) y en Medellín (1) se informa el caso de una paciente de 25 años con cáncer de endometrio y asociada con síndrome de ovario poliquístico.

Cuadro No. 1

GAMCER DE ENDOMETRIO

CLASIFICACION CLINICA

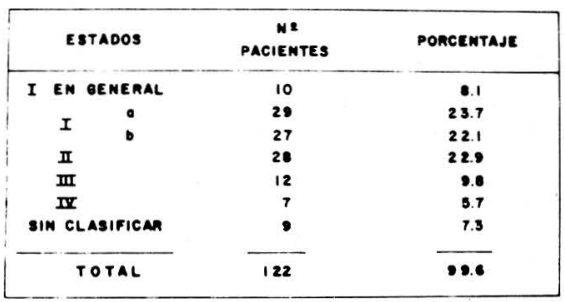

En el registro en cuanto a clasificación clínica encontramos que hay un $8.1 \%$ (o sea diez pacientes) que se clasifican como Estadio I de Endometrio sin que se haya consignado en forma detallada si es Estado I A ó I B pues en la historia muchas veces no aparece consignado el dato de la histerometría. Además, encontramos en $7.3 \%$ de pacientes sin clasificación clínica. No se consignó en la historia o fueron intervenidos particularmente y se remiten para tratamiento complementario con Radium o Cobalto.

Los porcentajes mayores fueron para los Estadios I A, I B - II respectivamente. Sin embargo, en este momento en la clasificación clínica se le da mucha importancia a la diferenciación histológica del tumor $y$ basado en este dato se podría hablar de pronóstico. En las historias analizadas sólo en algunos casos aparece registrado el grado de diferenciación histológica, por lo que no es posible establecer subgrupos en el Estadio I A y I B. A nivel mundial algunos informes dan cifras diferentes de sobrevida de acuerdo con subgrupos dependiendo de la histerometría, penetración miometrial y diferenciación histológica (4).

\section{Gráfica No. 2}

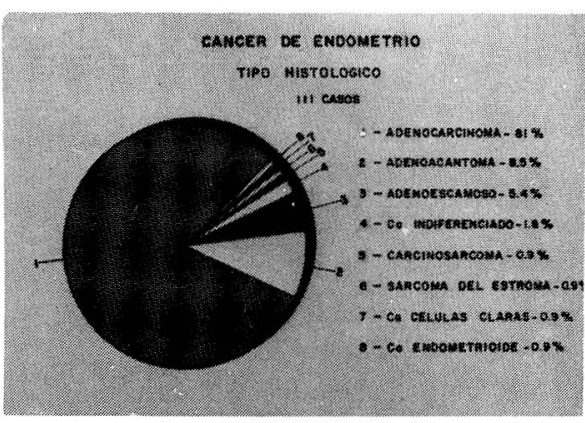

El tipo histológico aparece consignado en la historia en 111 casos $y$ predominantemente fue adenocarcinoma en, el $81 \%$ de los casos (90 pacientes). Luego aparecen en orden descendente el adenoacantoma, el adenoescamoso, etc. y aparecen casos tan raros como el carcinosarcoma y el sarcoma del estroma endometrial. En algunas descripciones microscópicas aparecen el informe detallado de la diferenciación celular, pero en otros el informe anatomopatológico es muy pobre y sólo se limita a diagnosticar adenocarcinoma endometrial. De todas formas es muy importante que el patológo informe el grado de diferenciación lo que contribuye a predecir el pronóstico.

Estas cifras de predominancia del adenocarcinoma endometrial no se apartan de los comunicados en la bibliografía internacional y a nivel nacional el estudio del doctor Jorge Zúñiga (2) de Cali analiza 61 casos en un período de 23 años, informa 83\% para el adecarcinoma endometrial y como dato curioso un 13응 para sarcoma endometrial que es un porcentaje considerable, teniendo en cuenta lo poco común de este tipo histológico. 
Gráfica No. 3

\section{DISTRIBUCION POR QUINQUENIOS}

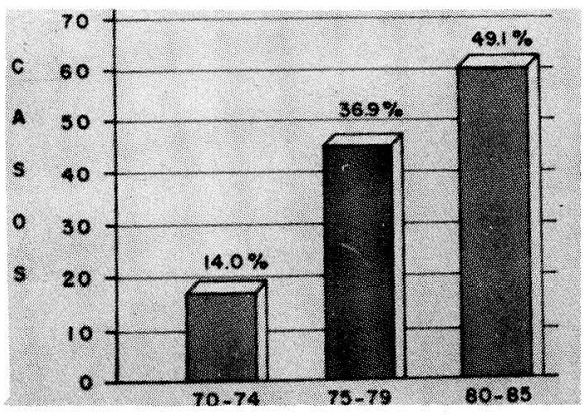

En la distribución del cáncer endometrial por quinquenios, encontramos una frecuencia creciente. Así vemos que en el quinquenio del 70-74 hubo 17 casos (14\%), en el quinquenio del 75-79 se registraron 45 casos (36.8응 y en el 80-85 el número de casos fue 60 (49.1\%) lo que está comprobando los datos de otros estudios a nivel mundial, que muestran una tendencia creciente del cáncer de endometrio. Faltaría por investigar o serviría como hipótesis de trabajo ver que factores intervienen en esta neoplasia: factores dietéticos, ambientales, niveles socio-económicos, etc.

\section{Gráfica No. 4}

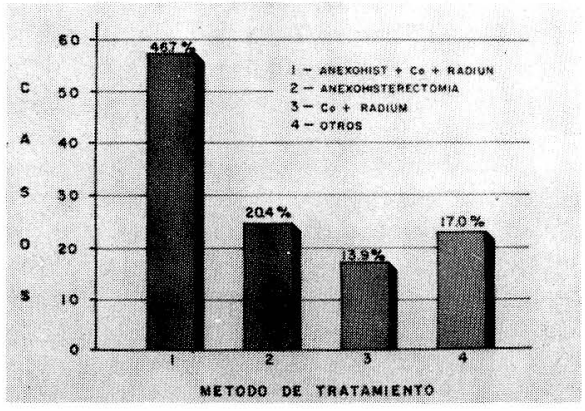

E! tratamiento efectuado en las pacientes comprende una gran variedad de modalidades terapéuticas. La decisión del tratamiento en cuanto a realizar primero Anexohisterectomía, luego Cobaltoterapia y Radium o viceversa se vio afectada en parte, por alguna complicación sobreagregada tal como obesidad extrema, edad avanzada, hipertensión no controlada.

Sin embargo, la mayoría de los casos siguió una línea clásica de tratamiento.

El porcentaje más elevado $46.7 \%$ es decir, 57 pacientes fueron tributarias de tratamiento triconjugado con base en Anexohisterectomía, Cobalto y Radium, en diferente orden bien sea iniciando con los medios actínicos, luego cirugía o viceversa. Actualmente, el tratamiento más reconocido es el quirúrgico y de acuerdo con el informe de patología se dará Radioterapia (4). El hecho de complementar con Cobalto se debió en parte al comprobar invasión profunda del miometrio por el tumor en algunos casos en los cuales el informe patológico fue explícito en este sentido.

Se realizó Anexohisterectomía solamente para los casos clasificados como I A o algunos I B, comprenden 25 pacientes o sea el $20.4 \%$.

En la gráfica el porcentaje titulado "otros tratamientos" incluye tres pacientes sometidas a Histerectomía Vaginal que además, de presentar su cáncer endometrial presentaban prolapso integral; tratamiento paliativo para pacientes con Estadio IV y Laparotomía por otra causa cuyo informe definitivo fue Adenocarcinoma de Endometrio y tratamientos hormonales con progestágenos.

Con relación al estudio histológico del especimen en la presente revisión, pudimos encontrar consignado en la historia, ochenta (80) muestras para un total de noventa y seis (6) operadas. Faltaron diez $y$ seis (16) estudios de patología, la muestra que presumimos 
sí fueron estudiados, pero no aparecen registrados en la historia. (Ver gráfica No. 5).

\section{Gráfica No. 5}

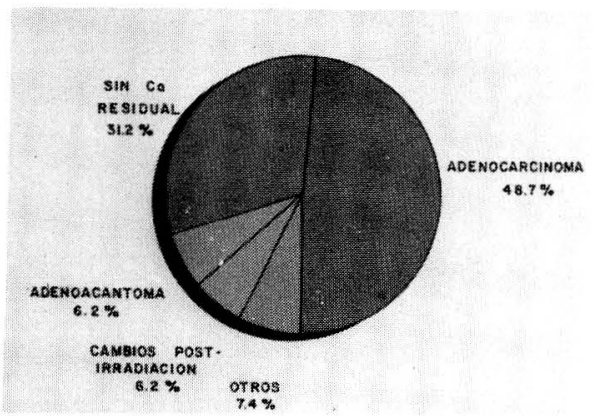

Otro dato importante hace referencia a muestras de pacientes que previamente habían sido irradiadas y en estos úteros no se encontró carcinoma residual. También se informó como cambios postirradiación. Este hecho nos hace suponer que en algunas pacientes si es de mucho valor el papel de la radioterapia previa, como tratamiento complementario del cáncer endometrial. A la luz de los conocimientos actuales es imperativo solicitar al patólogo el grado de invasión miometrial.

La Gráfica 6 correspondiente a seguimiento ilustra la distribución por quinquenios.

\section{Gráfica No. 6}

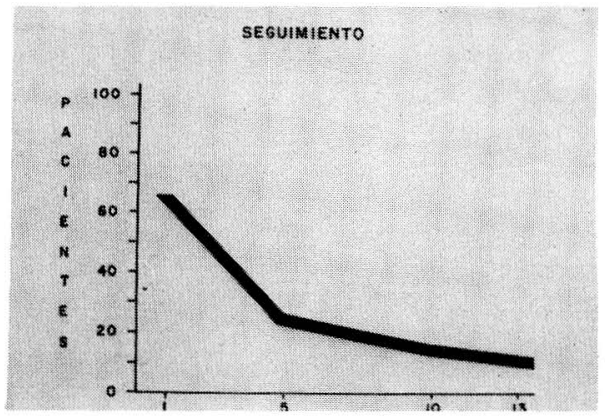

Un total de ochenta y ocho (8) pacientes tuvieron algún tipo de seguimiento Observamos que hay una tendencia a la declinación en el seguimiento a medida que transcurren los quinquenios; seguidas por más de cinco años había veinticinco (25) pacientes. En cambio seguidas por más de diez años se encontraron quince pacientes $y$ seguidas por más de trece años se encontraron diez pacientes. Es necesario hacer resaltar que el número absoluto de pacientes va disminuyendo, debido a que la edad de la misma es avanzada y posiblemente muchas de ellas mueren por otras causas distintas al tumor.

\section{Gráfica No. 7}

\section{Supervivencia}

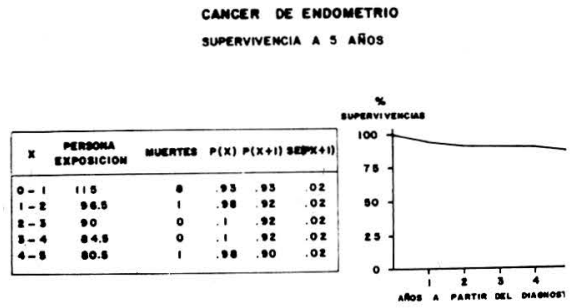

La supervivencia estimada por el método actual (11), (12), para las 122 pacientes cuyo diagnóstico fue cáncer de endometrio fue de $93 \%$ en el primer año de observación y de 98\% o más para los años segundo al quinto. La mayor mortalidad absoluta -ocho pacientes - se presenta también en el primer año; sólo dos muertes se registraron en los cuatro años siguientes. Al descontar las muertes y las pérdidas en el seguimiento se tenían ochenta (80) pacientes activas al finalizar el quinto año de seguimiento.

De este modo, la probabilidad acumulada de supervivencia desciende muy 
poco desde el 93\% en el primer año al $90 \%$ en el quinto año, tal como se aprecia en el gráfico adjunto.

\section{DISCUSION}

El riesgo en la mujer de padecer carcinoma endometrial es de 1 por mil por año y las que toman estrógenos, aumentan de 4 a 8 por 1.000 por año. En Cali (Colombia) la incidencia es de 7 por 100.000 y en los Estados Unidos es de $42 \times 100.000$.

La edad de aparición del cáncer endometrial clásicamente ha sido la quinta, sexta, séptima década de la vida, pero en la génesis de esta neoplasia hay algunos factores que pueden explicar el origen en las premenopáusicas $y$ en las postmenopáusicas.

En las Premenopáusicas: La Obesidad, el Síndrome de Ovario Poliquístico, Tumores productores de Estrógenos y la Terapia Hormonal Exógena desencadenarían estados de anovulación, que en forma crónica y mediante el efecto estrogénico persistente van a actuar sobre el órgano efector endometrial.

En épocas pasadas el uso de anovulatorios secuenciales se asociaron a la génesis del cáncer endometrial $(9,10)$. Actualmente, estos preparados en sus formas combinadas (Estrógeno - Progesterona) por el contrario, tienen efecto protector contra el cáncer endometrial

Esta secuencia de estados preneoplásicos a veces se puede interrumpir si corregimos los estados de anovulación crónica; se puede evitar este carcinoma, si se emprenden medidas para asegurar la ovulación (secreción de progesterona) o se da terapia combinada Estrógeno Progesterona (4). Ver grafica 8.
Gráfica No. 8

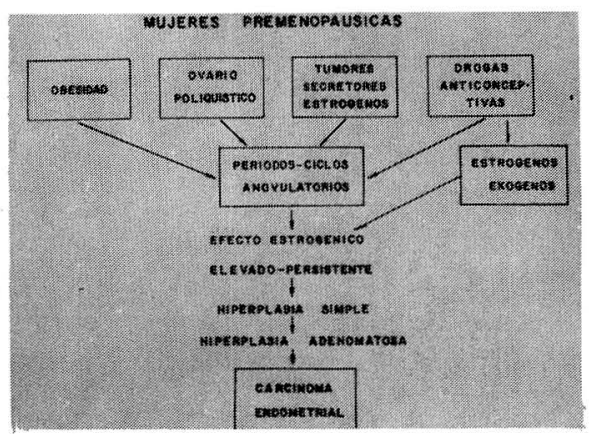

En las Postmenopáusicas: La función ovárica ha cesado pero. el efecto estrogénico bien sea exógeno o endógeno continúa siendo el desencadenante, que actuando en forma crónica y sostenida influencia directamente al órgano efector endometrial dando como resultado final el carcinoma. (Gráfica 9).

Gráfica No. 9

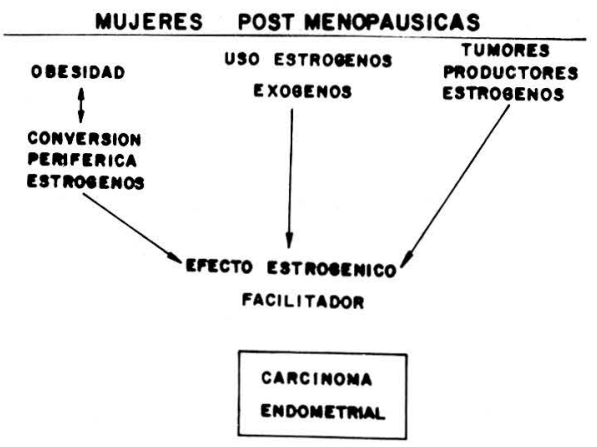

Estos dos fenómenos nos dan una visión general del origen del cáncer endome trial y de acuerdo con estas observaciones sobre la génesis del cancer endometrial, vemos que hay multitud de factores que llevan a un estado estro persistente $y$ en esta fase, en última instancia, es la estrona la hormona responsable directa de la génesis del carcinoma endometrial. 
Varios estudios consultados a nivel mundial $(3,4,5)$ hablan de factores pronósticos del cáncer endometrial o también llamados factores de riesgo. $\mathrm{Si}$ estos factores se tienen en cuenta tanto por parte del clínico como del patólogo, ayudarían a dar un mejor enfoque terapéutico y por lo tanto mejorarían las tasas de sobrevida.

\section{Estos factores son:}

1) Diferenciación Histológica.

2) Tamaño Uterino,

3) Estadio Clínico.

4) Invasión Miometrial.

5) Metástasis Ganglionar.

6) Citología Peritoneal.

7) Diseminación Anexial.

El pronóstico empeora: si el tumor es indiferenciado, si el tamaño uterino es mayor, el estadio clínico es avanzado, la penetración en el miometrio es más del $1 / 3$ interno y si hay metástasis ganglionares.

Al intervenir una paciente por cáncer de endometrio, se debe realizar lavado de cavidad peritoneal y la positividad de éste hablará también de mal pronóstico, lo mismo que la diseminación anexial. En nuestras historias estos datos no aparecen consignados y el informe anatomopatológico es pobre en descripción. Tampoco sabemos cuántas pacientes eran obesas, diabéticas, hipertensas o que padecian trastornos del ciclo, tenían miomatosis o pólipos endometriales, ovario poliquístico, o además tenían otro ca concomitante lo que nos induce a pensar en la necesidad de establecer un protocolo prospectivo de investigación para analizar estas variables.
Las mujeres con cáncer endometrial tienen factores epidemiológicos que la colocan en la categoría de personas de enorme riesgo de sufrir cáncer de mama. En nuestro estudio sólo se pudo constatar el caso de una paciente con cáncer de mama y endometrio.

Zúñiga (2) trae un cuadro donde analiza la patología asociada y nos muestra un $26 \%$ de asociación del cáncer endometrial con miomatosis, $22 \%$ de obesidad y $34 \%$ de hipertensión arterial.

En nuestro estudio estas variables fueron difíciles de evaluar pues no están consignadas en la historia clínica.

Todos estos indicadores deberían registrarse en un estudio futuro que permitan hacer un buen manejo del cáncer endometrial. Varios informes a nivel mundial $(6,7)$ hablan de la experiencia para tratamiento de casos avanzados del cáncer endometrial con tamoxifen (antiestrógeno ampliamente usado para cancer metastásico de mama en pacientes post-menopáusicas). Esto bloquearía el estímulo proliferativo del estrógeno. A nivel local la experiencia con esta droga específica para el cáncer endometrial es nula, lo que nos brinda la posibilidad de iniciar el manejo de pacientes con tumores endometriales usando el tamoxifen.

El tamoxifen sólo o en forma combinada con agentes alkilantes 0 antimetabolitos $(6,7,8)$ se ha utilizado como tratamiento paliativo del cáncer endometrial. Su efectividad se ha mostrado en pacientes que no responden a otros agentes, pero la respuesta más objetiva se aprecia en pacientes que tienen receptores estrógeno positivos. Se han obtenido respuestas significativas hasta $30 \%$ pero las objeciones mayores se deben al reducido número de pacientes. 


\section{CONCLUSIONES}

El número de casos del cáncer endometrial en el Hospital Universitario San Vicente de Paúl es considerable. Con relación al número total de casos de cáncer registrados en quince años (3.929), es aproximadamente (4.1\%).

La aparición por edades ocurre en los períodos comprendidos entre 40 y 70 años, es decir, en perimenopausia y postmenopausia.

El adenocarcinoma endometrial continúa siendo el tipo histológico más frecuente.

Los estadios iniciales hablan de un diagnóstico temprano que sería el ideal para asegurar un mejor pronóstico. EI tratamiento debe ser quirúrgico para ca- sos muy seleccionados es decir, para los estadios IA G1 y para otros estadios el tratamiento debe ser con Cobalto, Radium y Cirugía.

Se impone también la necesidad de obtener experiencia con drogas antiestrogénicas y progestágenos.

Se aprecia una tendencia el aumento de la frecuencia del carcinoma endometrial al evaluar el número de casos por quinquenios.

El número de casos seguidos de acuerdo con el número de años es considerable pero se debe mejorar más en el futuro. Es explicable que el seguimiento disminuya teniendo en cuenta la década tardía de aparición del carcinoma endometrial y la mortalidad de las pacientes por otras causas.

\section{ENDOMETRIAL CARCINOMA. FIFTEEN YEARS EXPERIENCE. HOSPITAL UNIVERSITARIO SAN VICENTE DE PAUL}

\section{CONCLUSIONS}

There is a significant number of cases of endometrial carcinoma at the Hospital Universitario San Vicente de Paul. With regard to the total number of cancer cases recorded in fifteen years (3.929) it accounts the (4.1\%) aproximately.

The age of appearance occurs between 40 and 70 years; i.e. in the perimenopausal and postmenopausal years.

Endometrial adenocarcinoma is still the most frequent histologic type.

The initial stages mean that an early Diagnosis should be the goal in acheiving a better prognosis. Treatment should be surgical for very selected cases; i.e, for stages $\mid A \mathrm{GI}$ and for the other stages, treatment should be Cobaltum, Radiumtherapy and surgery.

It is the imposed need to gain further experience with the use of antiestrogenic and progestational drugs.

A trend is seen to increase the frequency of endometrial cancer when evaluating the quinquenial number of cases.

The number of follow-up cases according to the number of years is significant, but must improve in the future. It is understood that the follow-up diminishes taking in account the late decade of appearance of the endometrial carcinoma and the mortality due to other causes in these patients. 


\section{BIBLIOGRAFIA}

1. URIBE GABRIEL, TOBON ALBERTO. Ca Endometrio en Pacientes Jóvenes. Rev. Col. Obst. Gin. Vol. XXXII No. 2, 137-139, 1981.

2. ZUÑLA JORGE, ZULUAGA FERNANDO. Tumores del Cuerpo Uterino. Rev. Col. Obst. Gin. Vol, XXXV No. 6: 465-472, nov.-dic. 1984.

3. TOONKEL L.M., JACOBSON L.H. Miometrial Penetration of Endometrial Carcinoma as a Prognostic Factor for Patients Receiving Pre or Postoperative Radiation Therapy. Am. J. Clin. Oncol. 7 (2): 113-4, 1984.

4. Hiperplasia y Neoplasia Endometriales. Clin. Obst. y Gin. Vol. 1, 1982.

5. CREASMAN W.T. Individualized Therapy for Endometrial Cancer. Symposium on Gynecol. Oncology, June 18, 1982. Berkeley California. Alto Bates Hospital.

6. KAUPPILA A. Endometrial Carcinoma Insensitive to Progestin and Cytotoxic. Chemotherapy May Respond to Tamoxifen. Acta Obst. Gynecol. Scand. 60 (6): 589-590, 1981.
7. QUINM MA. Tamoxifen and Aminoglutethimide in the Management of Patients with Advanced Endometrial Carcinoma not Responsive to Medroxiprogesterona, August No. 2 J. Obst. Gynecol. 21 (4): 226-229, 1981.

8. HALD I, Tamoxifen Treatment of Advanced Endometrial Carcinoma, A Phase II Study.

9. Conceptos actuales sobre anticonceptivos orales. Clínicas Obst. y Gin. Vol. 3, 1981.

10. SILVERBERG S. MAKOWSKI. Endometrial carcinoma in women under 40 year of age: comparison of cases in oral contraceptive users and Non - users Cancer 29: 592, 1977.

11. GREISS F.G., RHYNE L.A., LOCK F.R. The Role of Statistics in a Therapy Program for Gynaecological Cancer. Obstetrical and Gynaecological Survey, (20), 1965 ; pp. 603-618.

12. AXTELL, L.M. J. of the Am. Medical Association. 186, 13, Dec. 1963.

\title{
SEPTIMO CURSO $Y$ TERCER CONGRESO COLOMBIANOS DE MEDICINA GENERAL Y SOCIAL
}

\author{
Organizado por \\ ASOCIACION MEDICA DE ANTIOQUIA \\ Septiembre 25, 26 y 27 de 1986 -- MEDELLIN \\ Informes: $\quad$ AMDA. Carrera 50 No. 62-5 \\ Teléfonos: 2335391 y 2335547 \\ Anartado Aéreo 52578 - Medellín
}

